\begin{tabular}{|c|c|}
\hline Title & Turn-to-T urn Contact Resistance Measurement of No-Insulation REBCO Pancake Coil at DC Current Operation \\
\hline Author(s) & Noguchi, So; Tatsuta, Takahiro; Nishikawa, Daisuke; Ueda, Hiroshi; Kim, SeokBeom \\
\hline Citation & $\begin{array}{l}\text { IEEE transactions on applied superconductivity, 30(4), } 9000305 \\
\text { https://doi.org/10.1109/T A SC.2020.2970381 }\end{array}$ \\
\hline Issue Date & $2020-06$ \\
\hline Doc URL & http:/hdl.handle.net/2115/78149 \\
\hline Rights & $\begin{array}{l}\text { (c) } 2020 \text { IEEE. Personal use of this material is permitted. Permission from IEEE must be obtained for all other uses, in } \\
\text { any current or future media, including reprinting/republishing this material for advertising or promotional purposes, } \\
\text { creating new collective works, for resale or redistribution to servers or lists, or reuse of any copyrighted component of } \\
\text { this work in other works. }\end{array}$ \\
\hline Type & article (author version) \\
\hline File Information & FINAL_VERSION.pdf \\
\hline
\end{tabular}

Instructions for use 


\title{
Turn-to-turn Contact Resistance Measurement of No-Insulation REBCO Pancake Coil at DC Current Operation
}

\author{
So Noguchi, Takahiro Tatsuta, Daisuke Nishikawa, Hiroshi Ueda, and SeokBeom Kim
}

\begin{abstract}
No-insulation (NI) (RE) $\mathrm{Ba}_{2} \mathrm{Cu}_{3} \mathrm{O}_{y}$ (REBCO) magnets are very promising for practical applications to generate high magnetic field. The NI winding technique provides high thermal stability so that quench protection is unnecessary. However, the turn-to-turn contact resistance (sometimes called characteristic resistance) is an important factor to characterize the thermal stability and charging delay of NI REBCO pancake magnets. Although the conventional sudden-discharging method is widely used to measure the turn-to-turn contact resistance, it is not applicable to various conditions; e.g., the change of DC operating current and temperature.

Therefore, we have previously proposed a turn-to-turn contact resistance measurement method using a low-frequency AC (LFAC) current. In this paper, we measured the contact resistance for different DC operating currents or change of DC current to confirm the validity of LFAC method. In addition, by heating a test coil, the contact resistances were also measured during the change of DC operating current. Since the electromagnetic stress is small enough not to change the contact condition, the change in contact resistance is negligible. The measured contact resistance was unchanged, so we could conclude that the LFAC method is effective in measurement of the contact resistance when DC operating current was applied.
\end{abstract}

Index Terms-No-insulation winding technique, REBCO pancake coil, turn-to-turn contact resistance measurement.

\section{INTRODUCTION}

$\mathbf{T}$ OWARD practical rare-earth barium copper oxide (REBCO) applications, some REBCO magnets have been under development [1]-[3]. Since a normal zone in REBCO conductor slowly propagates, quench protection is very difficult. In 2011, Hahn et al. proposed the no-insulation (NI) winding technique to avoid burning-out REBCO magnets [4]. A radially bypassing current through relatively low resistance (often called "turn-to-turn contact resistance" or "characteristic resistance") provides high stability. Wang et al. were successful to measure a turn-to-turn contact resistance derived from a time constant of sudden-discharging tests [5]. Although the sudden-discharging method is effective in measurement of contact resistance, it is not applicable to various conditions;

Manuscript receipt and acceptance dates will be inserted here. (Corresponding author: So Noguchi.)

S. Noguchi is with the Graduate School of Information Science and Technology, Hokkaido University, Sapporo 060-0814, Japan (e-mail: noguchi@ssi.ist.hokudai.ac.jp).

T. Tatsuta, D. Nishikawa, H. Ueda, and S. Kim are with the Graduate School of Natural Science and Technology, Okayama University, Okayama 700-8530, Japan.

Color versions of one or more of the figures in this paper are available online at http://ieeexplore.ieee.org.

Digital Object Identifier will be inserted here upon acceptance. e.g., it is impossible to obtain the dependence of the contact resistance on a DC operating current or a temperature. In addition, the paper [6] showed that the contact resistance, and not the metal resistance, dominated the characteristic resistance of REBCO coils. That is, since an electromagnetic force working for NI pancake coil varies with the change of transport current and magnetic field, the contact resistance would also vary due to the change of the contact condition between REBCO tapes or metals.

Therefore, we have previously proposed a low-frequencyAC-current (LFAC) contact resistance measurement method [7]. The proposed LFAC method can measure the turn-toturn contact resistance of REBCO NI pancake coils under various conditions. In this paper, the contact resistances were measured carrying an operating DC current. In addition, during heating an NI REBCO pancake coil by heaters, the contact resistance was also measured. This paper shows the validity of the proposed low-frequency-AC-current contact resistance measurement method for the difference and change of DC operating current.

\section{LOW-FrEQUENCY-AC-CURRENT CONTACT Resistance Measurement Method}

As the detail of the low-frequency-AC-current contact resistance measurement method is shown in [6], the following condition must be held to measure the contact resistance:

$$
\alpha=\left|\frac{R_{\mathrm{ct}}}{j \omega L}\right| \ll 1
$$

where $\alpha, j, \omega, L$, and $R_{\mathrm{ct}}$ are the condition number, the imaginary unit, the current angular frequency, the coil inductance, and the turn-to-turn contact resistance, respectively. When the condition number $\alpha$ is much smaller than 1, the coil impedance $Z$ for the AC component of current is $R_{\mathrm{ct}}$ :

$$
Z=\frac{j \omega L R_{\mathrm{ct}}}{R_{\mathrm{ct}}+j \omega L}=R_{\mathrm{ct}}
$$

where $\left|R_{\mathrm{ct}}\right| \ll|j \omega L|$. As the contact resistance depends on the inductive impedance, it can be measured by applying an AC current of 1 to $50 \mathrm{~Hz}$ to an NI REBCO coil. It is also possible to measure it by superimposing an $\mathrm{AC}$ current on a $\mathrm{DC}$ transport current, because the $\mathrm{AC}$ impedance $Z$ corresponds to the contact resistance $R_{\mathrm{ct}}$. That is, the contact resistances are measured for different DC operating currents or during the change of operating current. 


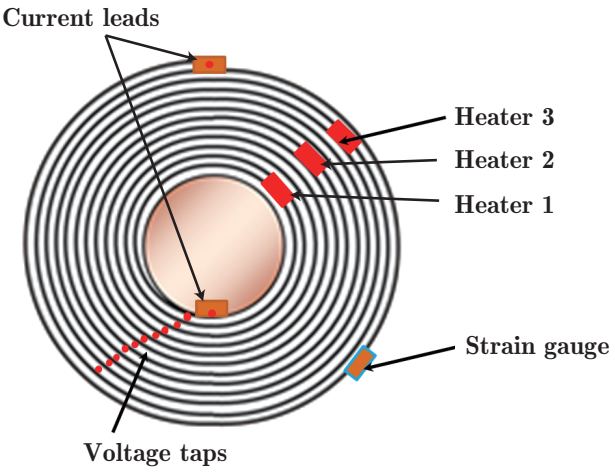

(a)

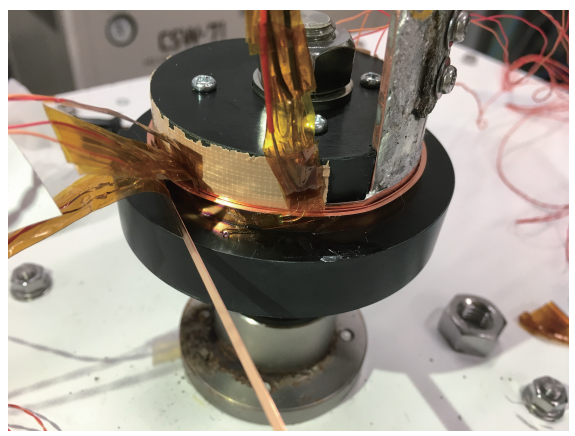

(b)

Fig. 1. (a) Schematic drawing of NI REBCO pancake coil with heaters and strain gauge. The Heaters 1, 2, and 3 are attached between 1st and 2nd turns, 5th and 6th, and 9th and 10th, respectively. (b) Photo of the tested NI REBCO pancake coil.

TABLE I

SPECIFICATIONS OF NI REBCO PANCAKE COIL

\begin{tabular}{ll} 
i.d.; o.d. [mm] & $60.0 ; 62.9$ \\
REBCO tape thickeness [mm] & 0.144 \\
REBCO tape width [mm] & 4 \\
Number of turns & 10 \\
Inductance $L[\mu \mathrm{H}]$ & 12.2 \\
\hline
\end{tabular}

\section{TURn-TO-TURn CONTACT RESISTANCE}

MEASUREMENT Under DiFFERENT DC CURRENTS

\section{A. Experimental conditions}

One NI REBCO pancake coil illustrated in Fig. 1(a) was tested for measurement of the contact resistance under different DC currents (the photo in Fig. 1(b)). Table I lists its specifications of the NI REBCO pancake coil. The NI REBCO pancake coil was wound at a tension of $1 \mathrm{~kg}$. The coil was cooled by immersion in liquid nitrogen. The contact resistances were measured at DC transport currents of 0 , 10,40 , and $80 \mathrm{~A}$. An $\mathrm{AC}$ current of $10 \mathrm{~A}$ at $10 \mathrm{~Hz}$ was superimposed on the DC transport currents. The computed coil inductance $L$ is $12.2 \mu \mathrm{H}$. According to (1) for $10-\mathrm{Hz}$ AC current, the contact resistance $R_{\mathrm{ct}}$ must be smaller than $765 \mu \Omega$ to obtain an accurate contact resistance.

\section{B. Experimental results and discussion}

Fig. 2 shows the measured waveforms at DC currents of 0, 10, 40, and 80 A. In Fig. 2, the DC + AC currents refer to raw measurement data, and the $\mathrm{AC}$ currents are obtained
TABLE II

CONTACT Resistances And Resistivities

\begin{tabular}{lcccc} 
DC current [A] & 0 & 10 & 40 & 80 \\
Contact resistance $R_{\mathrm{ct}}[\mu \Omega]$ & 142.6 & 143.9 & 146.3 & 146.3 \\
Contact resistivity $\rho_{\mathrm{ct}}\left[\mu \Omega \cdot \mathrm{cm}^{2}\right]$ & 122 & 123 & 125 & 125 \\
\hline
\end{tabular}

from subtracting the DC current component from them. However, the high-frequency components are eliminated. The AC impedances $Z$, i.e., the contact resistances $R_{\mathrm{ct}}$, for different DC currents are computed by the AC voltages divided by the AC currents. Table II shows the contact resistances and resistivities for different DC currents. Here, the contact resistivities $\rho_{\text {ct }}$ are obtained using the following relation [5]:

$$
R_{\mathrm{ct}}=\sum_{i=1}^{N-1} \frac{\rho_{\mathrm{ct}}}{2 \pi r_{i} w}
$$

where $r_{i}$ and $w$ are the radius of $i$ th turn and REBCO tape width, respectively.

Fig. 3 also shows the relation of contact resistance and DC current. It can be seen that although there was a slight difference of the contact resistance for different DC currents, almost the same values were obtained.

Next, we investigated the contact resistance dependence of the radial stress. Fig. 4 shows the radial stress computed using the finite element method [8]. Fig. 5 presents the constant resistance as a function of the minimum radial stress. From Fig. 5, it is found out that the contact resistances are almost constant because the radial stresses are too small to change the contact condition between turns.

Due to a very small electromagnetic force, the contact condition between turns was unchanged. Hence, we concluded that it was successful to measure the turn-to-turn contact resistance, even though a DC operating current was applied. Although the sudden-discharging method cannot apply an NI REBCO pancake coil with DC current, the proposed LFAC method reveals a potential to measure under various conditions.

\section{Contact Resistance Measurement during HEATING}

To investigate the further validity of the proposed lowfrequency-AC-current contact resistance method, we measured the contact resistance while the NI REBCO pancake coil was being heated by the Heater 1 or 2 as drawn in Fig. 1. An energy of $100 \mathrm{~J}$ was injected to the coil for $10 \mathrm{~s}$, when a DC current of 80 A carries. A 10-A sinusoidal current of $10 \mathrm{~Hz}$ is superimposed to measure the contact resistance. Fig. 6(a) shows the waveform of the coil voltage, the AC current, and the $\mathrm{DC}+\mathrm{AC}$ current in the case of Heater 1 between 1 st and 2 nd turns. The obtained contact resistances at 2, 5, 10, 15, and $18 \mathrm{~s}$ are shown in Fig. 6(b) and Table III. The contact resistance during heating slightly increases, but is almost unchanged. FIg. 6(c) presents the center magnetic field. During heating, the center magnetic field is not greatly changed. However, since the coil voltage rose, a local normal zone appeared around Heater 1 and the azimuthal current was induced in the outer turns in the superconducting state, 


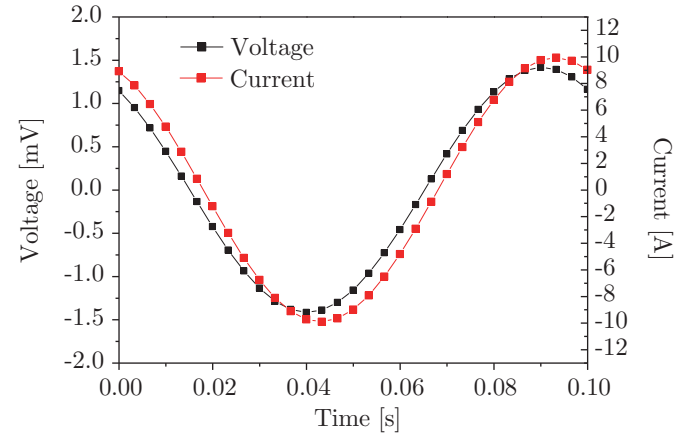

(a)

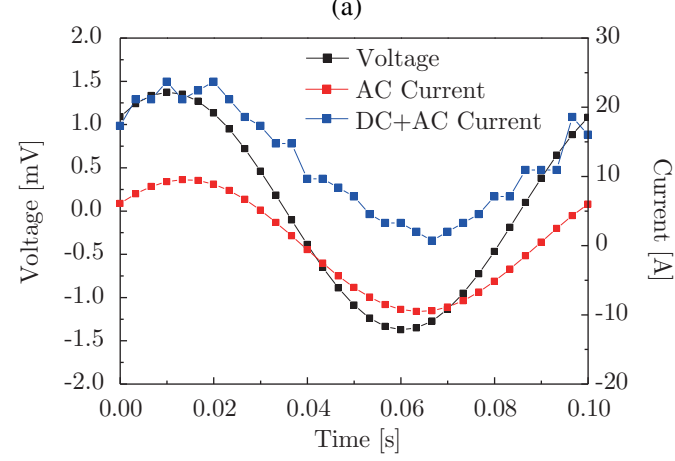

(b)

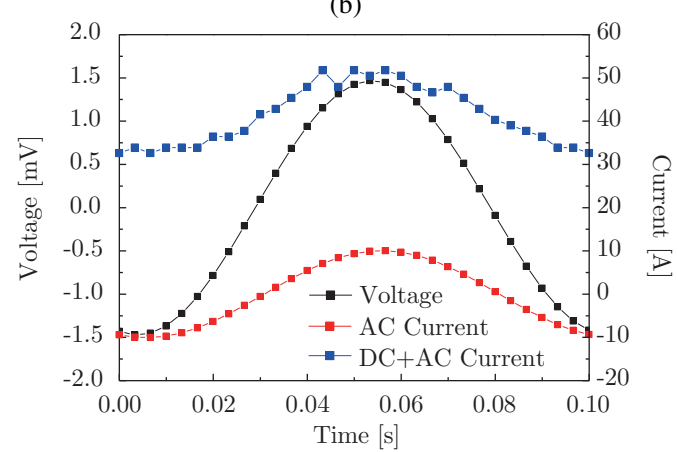

(c)

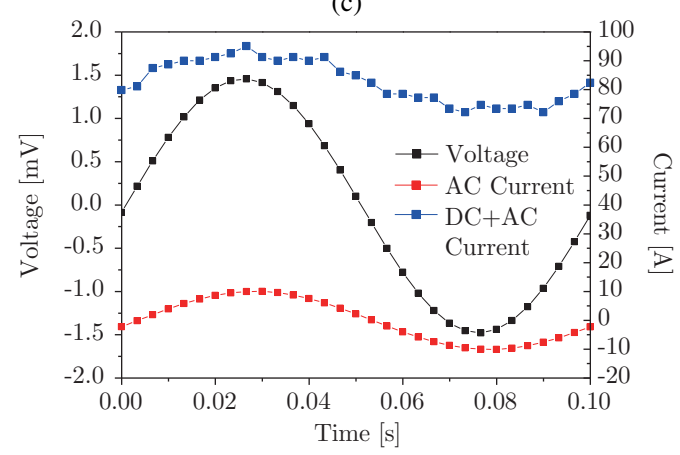

(d)

Fig. 2. Measurement coil voltage, $\mathrm{DC}+\mathrm{AC}$, and $\mathrm{AC}$ currents at $\mathrm{DC}$ current of (a) 0, (b) 10, (c) 40, and (d) $80 \mathrm{~A}$. The $\mathrm{AC}+\mathrm{DC}$ current waveforms are raw measurement data. The $\mathrm{AC}$ currents were obtained from subtracting DC component from the raw AC + DC current data, and high-frequency components were removed.

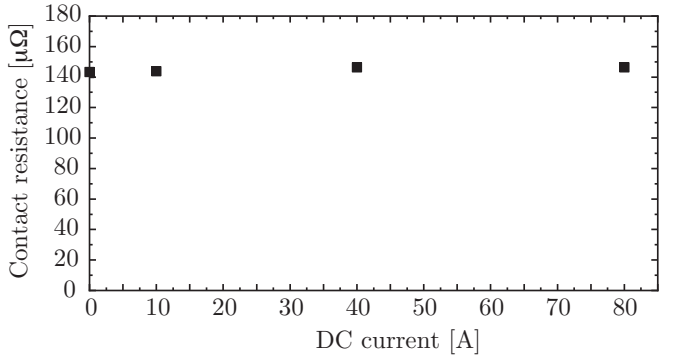

Fig. 3. Contact resistance dependence of DC current.

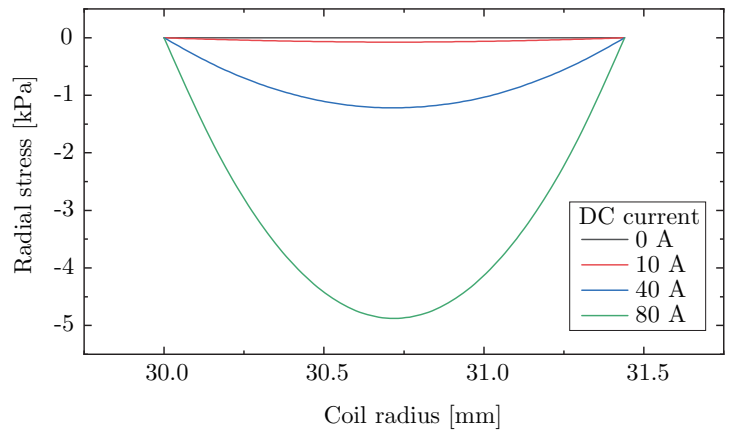

Fig. 4. Radial stress of NI REBCO pancake coil.

so that the center magnetic field kept. Since the constant contact resistance is obtained despite the occurrence of the local normal zone, the proposed contact resistance method enables measurement during heating.

Next, the NI REBCO coil was heated by Heater 2 located between the 5th and 6th turns. The heat amount and duration were the same as Heater 1. Fig. 7(a) also presents the coil voltage, the AC component, and the $\mathrm{DC}+\mathrm{AC}$ current. Fig. 7(b) and Table IV shows the contact resistance $R_{\text {ct }}$ as time passes. The center magnetic field is plotted in Fig. 7(c). It largely dropped during heating, and then the contact resistance decreased. However, since it was a slight decrease, it was successful to correctly measure the contact resistance in the case of Heater 2, too.

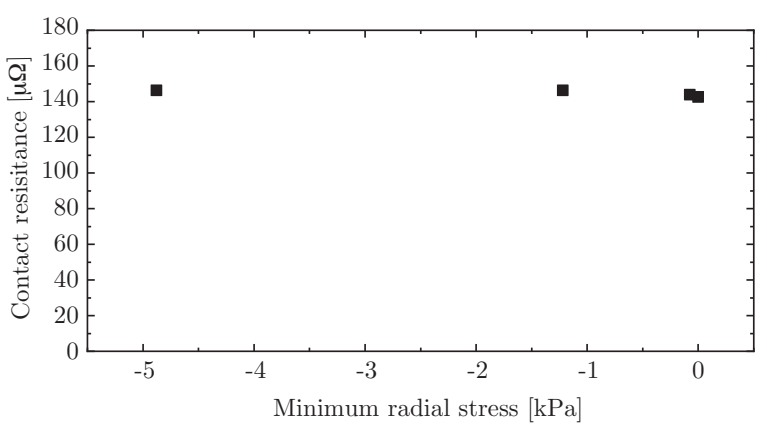

Fig. 5. Contact resistance as a function of minimum radial stress.

TABLE III

Contact Resistances During Heating (Heater 1)

\begin{tabular}{llllll}
\hline Time [s] & 2 & 5 & 10 & 15 & 18
\end{tabular}

\begin{tabular}{llllll} 
Contact resistance $[\mu \Omega]$ & 161.6 & 170.9 & 179.9 & 174.5 & 155.4 \\
\hline
\end{tabular} 


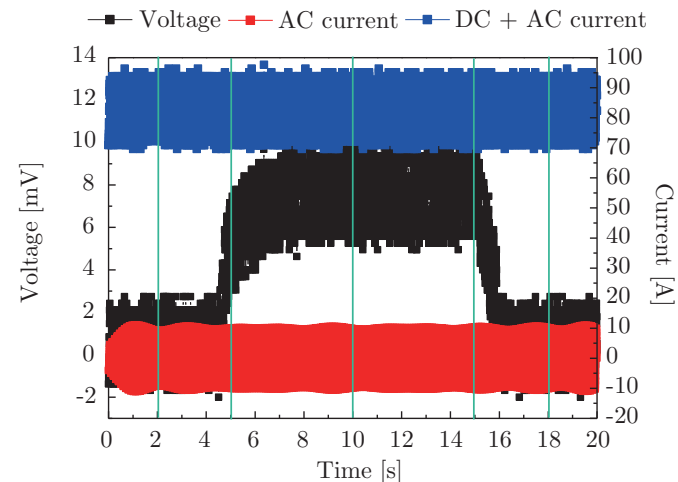

(a)

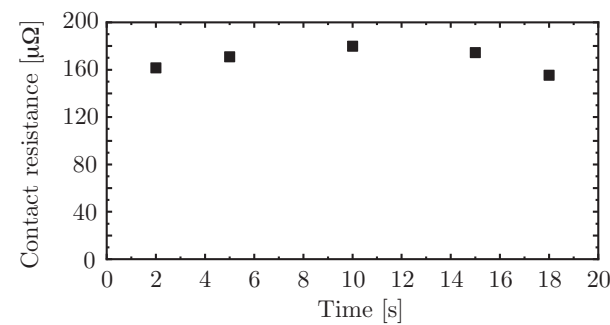

(b)

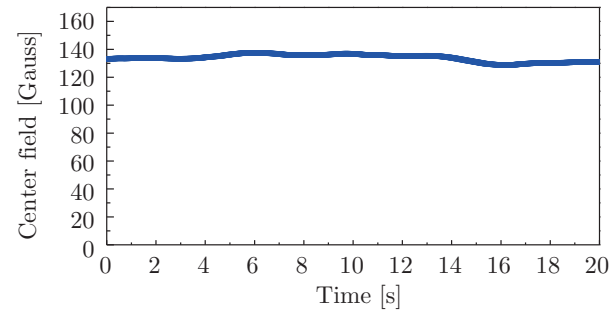

(c)

Fig. 6. (a) waveforms of coil voltage, $\mathrm{AC}$ component, and $\mathrm{DC}+\mathrm{AC}$ current, (b) contact resistance, and (c) magnetic field at coil center during heating by Heater 1.

TABLE IV

Contact Resistances During Heating (Heater 2)

\begin{tabular}{lccccc}
\hline Time $[\mathrm{s}]$ & 2 & 5 & 10 & 16 & 18 \\
Contact resistance $[\mu \Omega]$ & 153.3 & 155.0 & 128.6 & 145.2 & 165.2 \\
\hline
\end{tabular}

The NI coil behavior during heating is very complicated. When the current distribution changes, the stress inside the coil also changes. In addition, when the temperature increases, the stress distribution also changes due to the thermal strain. However, the contact condition between REBCO tapes was unchanged in these experiments, because the stress change was very small. Therefore, almost constant contact resistances were obtained. Since the unchanged values were obtained, we can conclude that the proposed LFAC method is very robust under a condition change.

In the near future, we will apply the LFAC method to the measurement of changing contact resistance; e.g., a large external field is applied to an NI REBCO pancake coil. The results will be given together with a simulation result.

\section{CONClusion}

For practical no-insulation (NI) REBCO magnet applications, it is necessary to clarify the turn-to-turn contact resistance of NI pancake coil under various conditions. However,

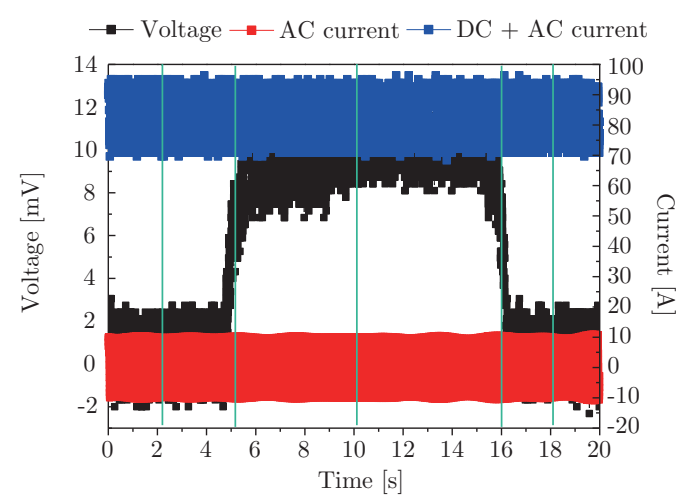

(a)

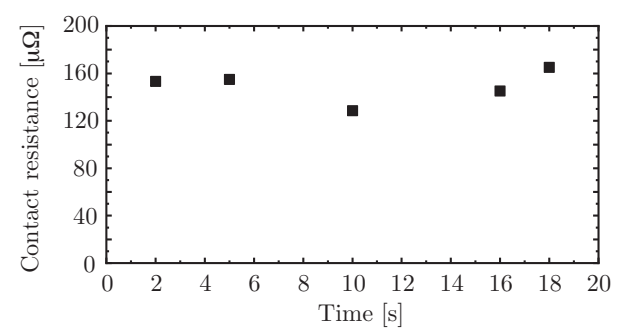

(b)

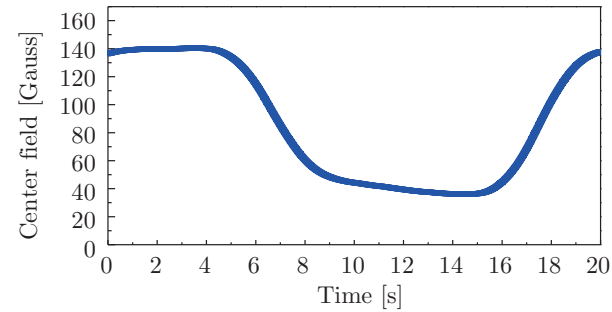

(c)

Fig. 7. (a) waveforms of coil voltage, AC component, and DC + AC current, (b) contact resistance, and (c) magnetic field at coil center during heating by Heater 2.

the application of the conventional sudden-discharging method is very limited. Hence, we have previously proposed a lowfrequency-AC-current (LFAC) method to measure the turnto-turn contact resistance of NI REBCO pancake coil, and compared the measurement results with those of the suddendischarging method to confirm the validity.

In this paper, the contact resistances under different DC current operation or during heating the NI REBCO coil were measured. Since the stress of actual contact was small enough not to change the contact condition of REBCO tapes, the unchanged contact resistance was measured. As a result, we can conclude that the proposed LFAC method is effective in the conditions of DC current and its change.

In the near future, we will measure the turn-to-turn contact resistance under different contact conditions; the change of the temperature and external field. And then, the feature of turn-to-turn contact resistance will be clarified further.

\section{REFERENCES}

[1] S. Hahn, K. Kim, K. Kim, X. Hu, T. Painter, I. Dixon, S. Kim, K. R. Bhattarai, S. Noguchi, J. Jaroszynski, and D. C. Larbalestier, "45.5tesla direct-current magnetic field generated with a high-temperature superconducting magnet," Nature, vol. 570, pp. 496-499, Jun. 2019. 
[2] H. Ueda, M. Fukuda, K. Hatanaka, T. Wang, X. Wang, A. Ishiyama S. Noguchi, S. Nagaya, N. Kashima, and N. Miyahara, "Conceptual Design of Next Generation HTS Cyclotron," IEEE Trans. Appl. Supercond., vol. 23, no. 3, Jun. 2013, Art. no. 4100205.

[3] Y. Iwasa, J. Bascuñán, S. Hahn, J. Voccio, Y. Kim, T. Lécrevisse, J. Song, and K. Kajikawa, "A high-resolution 1.3-GHz/54-mm LTS/HTS NMR magnet," IEEE Trans. Appl. Supercond., vol. 25, no. 3, Jun. 2015, Art. no. 4301205.

[4] S. Hahn, D. K. Park, J. Bascuñán, and Y. Iwasa, "HTS pancake coils without turn-to-turn insulation," IEEE Trans. Appl. Supercond., vol. 21, no. 3, pp. 1592-1595, Jun. 2011.

[5] X. Wang, S. Hahn, Y. Kim, J. Bascuñán, J. Voccio, H. Lee, and Y. Iwasa, "Turn-to-turn contact characteristics for an equivalent circuit model of no-insulation ReBCO pancake coil," Supercond. Sci. Technol., vol. 26, no. 3, Mar. 2013, Art. no. 035012.

[6] S. Noguchi, K. Monma, H. Igarashi, and A. Ishiyama, "Investigation of Current Flow Between Turns of NI REBCO Pancake Coil by 2-D FiniteElement Method," IEEE Trans. Appl. Supercond., vol. 26, no. 3, Apr. 2016, Art. no. 4901205.

[7] S. Noguchi, R. Miyao, H. Okusa, T. Tatsuta, H. Ueda, and S. Kim, "Turn-to-turn Contact Resistance Measurement of No-Insulation REBCO Pancake Coils," IEEE Trans. Appl. Supercond., vol. 29, no. 5, Aug. 2019, Art. no. 4601605.

[8] S. Noguchi, "Electromagnetic, Thermal, and Mechanical Quench Simulation of NI REBCO Pancake Coils for High Magnetic Field Generation," IEEE Trans. Appl. Supercond., vol. 29, no. 5, Aug. 2019, Art. no. 4602607. 